\title{
Multiple Metastasizing Pleomorphic Adenomas of the Lung
}

\author{
Ayaka Nakai ${ }^{1}$, Kensuke Suzuki ${ }^{1}$, Hideaki Furuse ${ }^{1}$, Takeshi Tsuda ${ }^{1}$, Yasuaki Masaki ${ }^{1}$, \\ Hideki Shinno $^{2}$, Yoshitaka Ito ${ }^{2}$, Hideki Miyazawa ${ }^{2}$ and Hirokazu Taniguchi ${ }^{1}$
}

\begin{abstract}
A 40-year-old woman was referred to our hospital with abnormal findings on chest X-ray. Her medical history was remarkable in that she had presented with a pleomorphic adenoma in the right parotid gland treated by surgical removal approximately 12 years previously. Chest computed tomography showed well-defined non-calcified nodules of the bilateral lobes, so she underwent segmentectomy of the right upper lobe and middle lobe. The histopathological diagnosis was metastasizing pleomorphic adenoma of the lung, a rare entity.
\end{abstract}

Key words: pleomorphic adenoma, lung metastasis, parotid tumor

(Intern Med 56: 691-694, 2017)

(DOI: 10.2169/internalmedicine.56.7596)

\section{Introduction}

Pleomorphic adenoma is the most common benign neoplasm of the salivary glands $(1,2)$. Pleomorphic adenomas rarely metastasize without a histological malignant transformation (2-4). However, due to the rarity of such events, there is no clear consensus regarding their epidemiology, mechanism of development, course, treatment, prognosis, or survival. This case report describes the diagnostic approach for a patient with multiple metastasizing pleomorphic adenomas of the lung.

\section{Case Report}

A 40-year-old woman was referred to our hospital with abnormal findings on chest X-ray. Her medical history was remarkable in that she had had a pleomorphic adenoma in the right parotid gland treated with enucleation in our hospital approximately 12 years prior. That tumor had been $3.6 \times$ $3.2 \mathrm{~cm}$ in diameter, and while partial extracapsular growth and invasion of the capsular blood vessels were observed, there had been no cervical lymph node metastasis.

A chest X-ray revealed bilateral multiple nodules (Fig. 1).

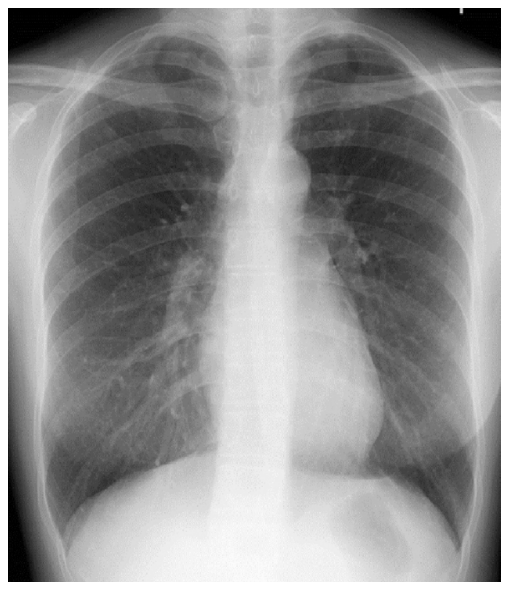

Figure 1. A chest X-ray at the first visit showed bilateral multiple nodules.

A chest computed tomography (CT) scan showed welldefined non-calcified nodular lesions of the bilateral lobes, with a maximum diameter of $11 \mathrm{~mm}$ (Fig. 2). 18-fluoro-2deoxyglucose positron emission tomography (FDG-PET) showed slightly positive signals (SUVmax: 1.0) in multiple lung nodules. For a diagnosis, two nodules of the right upper lobe and one nodule of the right median lobe were par-

${ }^{1}$ Department of Internal Medicine, Toyama Prefectural Central Hospital, Japan and ${ }^{2}$ Department of Thoracic Surgery, Toyama Prefectural Central Hospital, Japan

Received for publication April 16, 2016; Accepted for publication July 6, 2016

Correspondence to Dr. Kensuke Suzuki, khiro.tm@gmail.com 

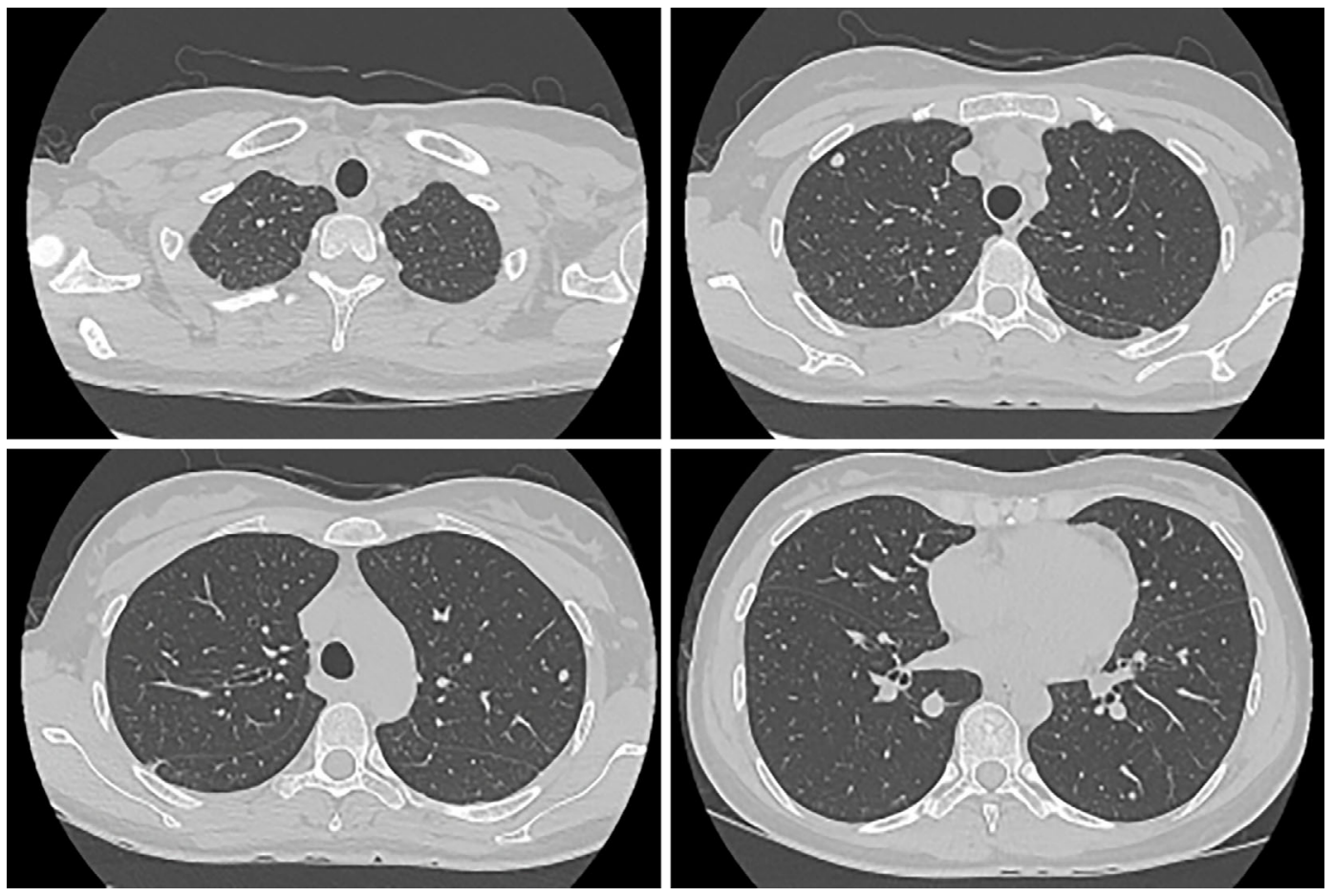

Figure 2. Chest CT at the first visit showed well-defined non-calcified nodular lesions of the bilateral lobes.
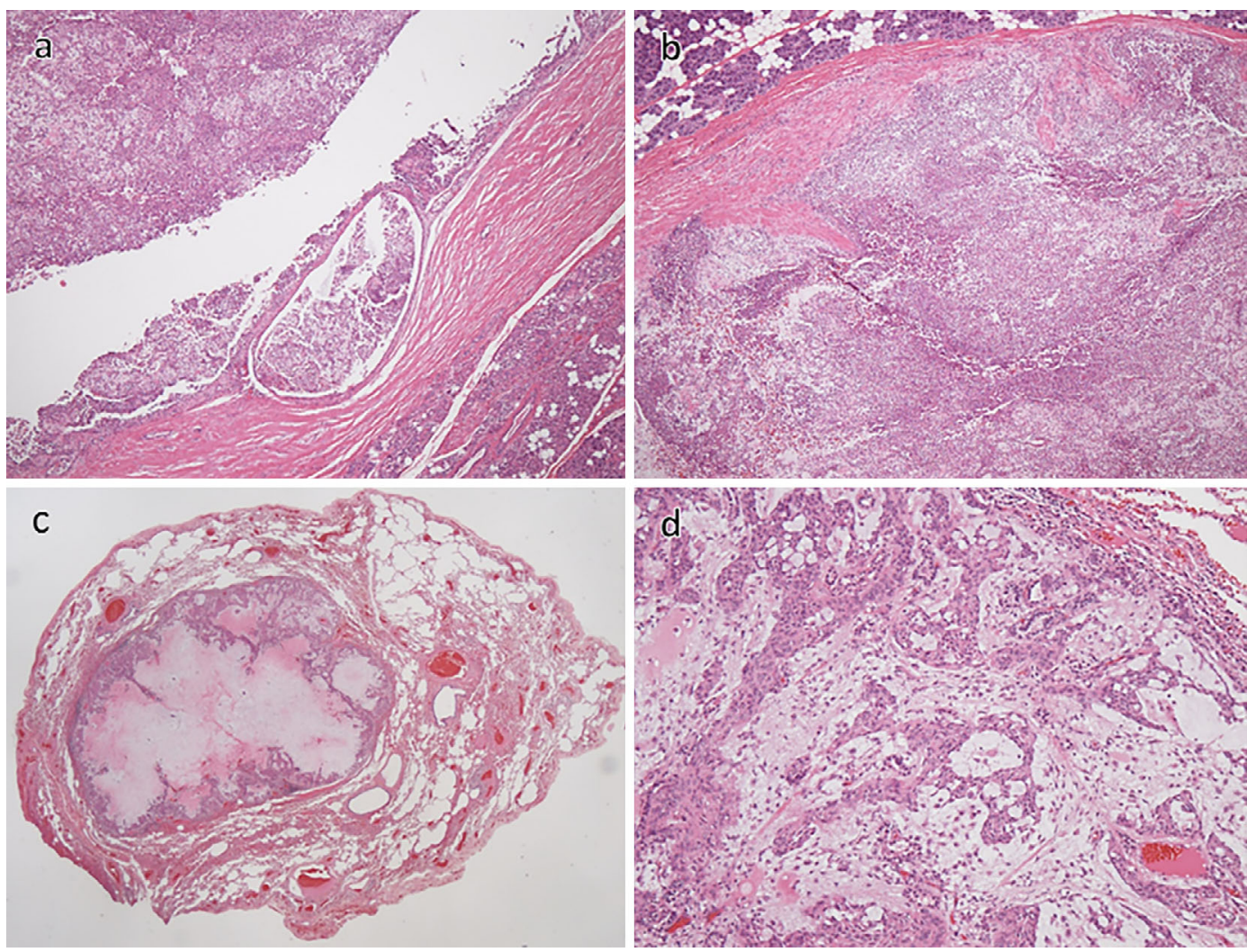

Figure 3. (a) A surgical specimen of the parotid gland showing tumor cells, consisting of epithelial and mesenchymal cells, and venous invasion [Hematoxylin and Eosin (H\&E) staining, 4×magnification]. (b) Tumor cells invading into subcapsular spaces in the parotid gland (H\&E staining, 4×magnification). (c) A surgical specimen of lung showing a well-demarcated whitish solid nodule (H\&E staining, 1 $\times$ magnification). (d) Tumor cells in the metastatic lesion consisting of epithelial and mesenchymal cells (H\&E staining, 10×magnification). 

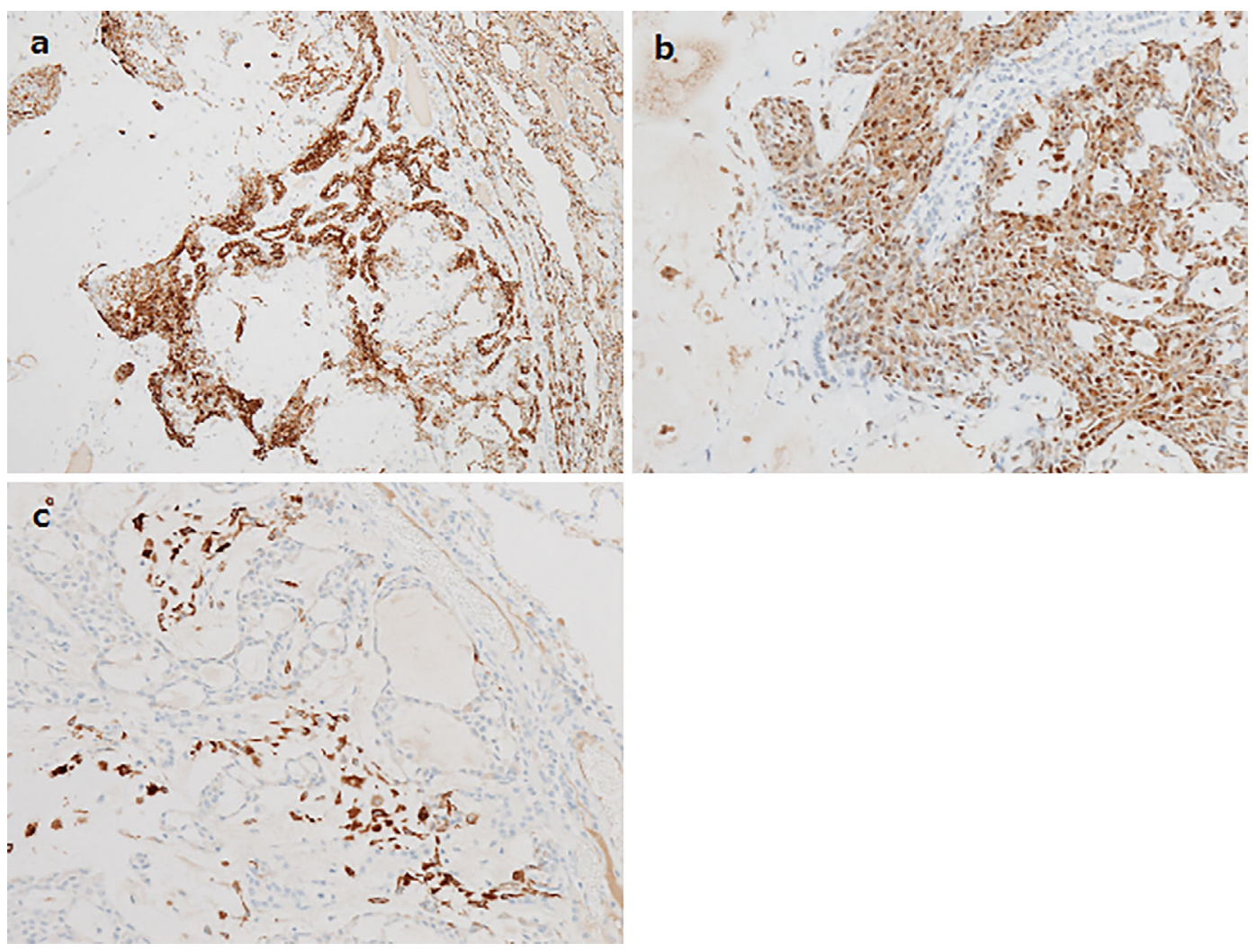

Figure 4. (a) Immunohistochemistry showing positivity for pan-cytokeratin AE1/AE3 in the epithelial cells and negativity in the mesenchymal cells (10xmagnification). (b) Immunohistochemistry showing positivity for S-100 protein in the epithelial cells (20×magnification). (c) Immunohistochemistry showing positivity for glial fibrillary acidic protein (GFAP) in the mesenchymal cells (20×magnification).

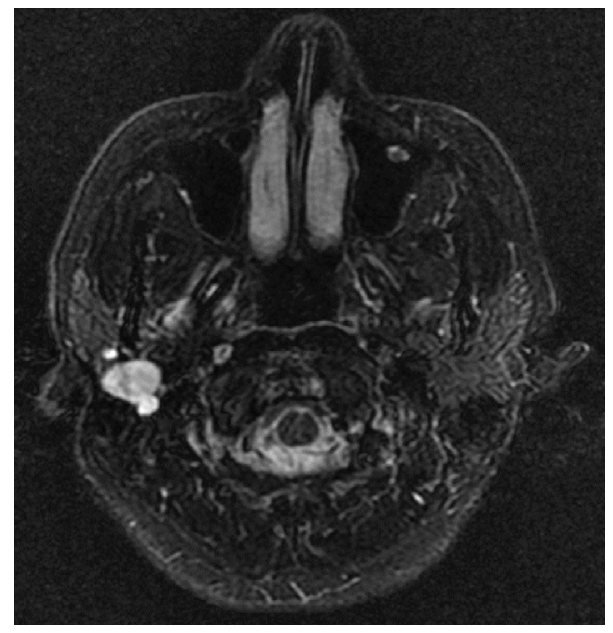

Figure 5. Contrast-enhanced MRI (T2WI) showed a 17-mm tumor in the right parotid gland.

tially resected by thoracoscopy. A macroscopic examination showed well-demarcated whitish solid masses with surfaces that were not smooth. A histological examination showed that the tumors contained a central epithelial component with proliferating polygonal or fusiform epithelial cells without nuclear atypia, and a peripheral mesenchymal component with loose fusiform cells in a myxoid stroma. In the surgical specimen of the parotid gland obtained 12 years previously, the tumor cells exhibited vascular and subcapsular invasion. These pathological findings were highly similar to the current findings, and thus the tumors were considered to have the same origin (Fig. 3).

An immunohistochemical analysis was positive for pancytokeratin AE1/AE3 and S-100 protein in the epithelial cells. The mesenchymal cells were negative for AE1/AE3 and positive for glial fibrillary acidic protein (GFAP) in the border zone between the epithelial and mesenchymal cells (Fig. 4). The MIB-1 labeling index (L.I.) was less than $3 \%$. The final diagnosis was metastasizing pleomorphic adenoma. Supporting the theory that the new tumor represented a local recurrence of the previous parotid gland tumor, a 17$\mathrm{mm}$ section of the right parotid gland tumor was examined by contrast-enhanced magnetic resonance imaging (MRI) (Fig. 5).

Resection of the local recurrence was likely to cause facial nerve paralysis, so this patient is being followed closely without treatment. In the three months since the diagnosis, enlargement of the local recurrence site and metastasis have not been observed.

\section{Discussion}

Foote and Frazell first reported a case of metastasizing pleomorphic adenoma in 1953 (5). Ratnasingham reviewed 
81 cases reported from 1942 to 2014 in the English language literature (2) and found that the most common sites for metastasizing pleomorphic adenoma were bone (36.6\%), lung $(33.8 \%)$, and cervical lymph nodes $(20.1 \%)$ and that the local recurrence rate prior to metastasizing pleomorphic adenoma was $72.8 \%$ (2). In our case, bone and cervical node metastases were not identified by whole-body contrast enhanced CT or FDG-PET.

The World Health Organization (WHO) classifies malignancies originating from pleomorphic adenomas into three forms of malignant epithelial tumors: carcinoma expleomorphic adenoma, carcinosarcoma, and metastasizing pleomorphic adenoma (6). Metastasizing pleomorphic adenoma is distinguished from the two other categories by its benign histology, and our case is consistent with this category. However, as metastasis is pathologically a feature of malignancy, metastasizing pleomorphic adenoma is an oxymoron.

The mechanism by which pleomorphic adenomas metastasize is poorly understood. Thackray and Lucas explained that this metastasis occurs by the "transplantation" mechanism based on the high rates of local recurrence, seeding, and tumor embolisms in capsular blood vessels, as well as local recurrence prior to metastasis (7). Inappropriate surgical resection is thought to allow disrupted tumor cells to be seeded through the venous or lymphatic routes (8). Nouraei revealed that incomplete surgical excision of the primary metastasizing pleomorphic adenoma was associated with local recurrence and distant metastasis (9). As the tumor in our case exhibited invasion of capsular blood vessels, surgical excision might have led to seeding.

Total surgical resection is the standard therapy of local recurrence and metastasis. Indeed, surgical metastasectomy confers a significant survival advantage over non-surgical treatment (9). Some patients have been reported to receive chemotherapy and radiotherapy, but there are no established treatments. The prognosis for metastasizing pleomorphic adenoma is difficult to quantify because such patients have not been subject to long-term follow-up, and case reports have often been published relatively early after treatment. The 1-year survival rate has been reported to be $80.4 \%$, and the 2-, 3-, and 5-year survival rates have been reported to be $64.7 \%, 41.2 \%$, and $31.4 \%$ (2). The independent prognostic factors are that the metastases are present at a signal site and that the interval between the initial primary tumor pres- entation and distant metastasis is more than 10 years; the 4year survival with the former and the latter are reportedly more than $70 \%$ and more than $80 \%$ (9). The clinical course of this patient meets these two factors, so the prognosis may be relatively promising.

In conclusion, we herein reported a rare case of metastatic pleomorphic adenoma of the lung. In pleomorphic adenoma patients, long-term follow-up is mandatory after resection due to the possibility of recurrence and metastasis.

The authors state that they have no Conflict of Interest (COI).

\section{Acknowledgement}

We thank Dr. Akio Uchiyama and Dr. Shin Ishizawa, the Department of Pathology, Toyama Prefectural Central Hospital, for providing the pathological images.

\section{References}

1. Gakidis I, Mihos PT, Chatziantoniou C, et al. A large neglected pleomorphic adenoma of the lung: Report of a rare case. Asian Cardiovasc Thorac Ann 22: 620-622, 2014.

2. Knight J, Ratnasingham K. Metastasising pleomorphic adenoma: Systematic review. Int J Surg 19: 137-145, 2015.

3. Kessoku H, Yoshimura T, Iino T, et al. A case of parotid pleomorphic adenoma metastasis to multiple organs. Nihon Jibiinkoka Gakkai Kaiho 117: 46-51, 2014 (in Japanese, Abstract in English).

4. Rodríguez-Fernández J, Mateos-Micas M, Martínez-Tello F. J., et al. Metastatic benign pleomorphic adenoma. Report of a case and review of the literature. Med Oral Patol Oral Cir Bucal 13: E193E196, 2008.

5. Foote FW, Frazell EL. Tumors of the major salivary glands. Cancer 6: 1065-1133, 1953.

6. Barnes L, Eveerson JW, Reichaet P, Sidransky D. Pathology and Genetics of Head and Neck Tumours. Barnes L, Eveerson JW, Reichaet P, Sidransky D, Eds. IARC Press, Lyon, 2005: 209-282.

7. Thackray AC, Lucas RB. Tumors of major salivary glands. In: Atlas of tumor pathology, snd series, Fascicle 10. Thackray AC, Lucas RB, Eds. Armes Forces Institute of Pathology, Washington DC, 1974: 16-39.

8. Sabesan T, Ramchandani PL, Hussein K. Metastasising pleomorphic adenoma of the parotid gland. Br J Oral Maxillofac Surg 45: 65-67, 2007.

9. Nouraei SAR, Ferguson MS, Clarke PM, et al. Metastasizing pleomorphic salivary adenoma. Arch Otolaryngol Head Neck Surg 132: 788-793, 2006.

The Internal Medicine is an Open Access article distributed under the Creative Commons Attribution-NonCommercial-NoDerivatives 4.0 International License. To view the details of this license, please visit (https://creativecommons.org/licenses/ by-nc-nd/4.0/).

(C) 2017 The Japanese Society of Internal Medicine http://www.naika.or.jp/imonline/index.html 